\title{
AGN and the Demographics of Supermassive Black Holes
}

\author{
Richard F. Green ${ }^{1,2}$ \\ Kitt Peak National Observatory, Tucson, AZ, USA; rgreen@noao.edu \\ ${ }^{1}$ On behalf of the STIS Instrument Definition Team Galaxy Nuclei \\ Group: Gary Bower, John Hutchings, Charles Joseph, Mary Elizabeth \\ Kaiser, John Kormendy, Charles Nelson, Douglas Richstone, Donna \\ Weistrop, Bruce Woodgate. \\ ${ }^{2}$ On behalf of the Nuker Team: Gary Bower, Alan Dressler, Sandra \\ Faber, Alex Filippenko, Karl Gebhardt, Carl Grillmair, Luis Ho, John \\ Kormendy, Tod Lauer, John Magorrian, Jason Pinkney, Douglas \\ Richstone, Christos Siopis, Scott Tremaine.
}

\begin{abstract}
.
High angular resolution observations from WFPC and STIS now allow well-constrained dynamical measurement of the masses of supermassive black holes (SMBH) in nearby galaxies. An initial statistical analysis by Magorrian et al. showed that $97 \%$ of bulges host SMBH. Black hole mass is correlated moderately with bulge luminosity and strongly with the velocity dispersion of the whole bulge, suggesting that black hole formation may be an intrinsic aspect of bulge formation. Black hole masses for AGN determined from reverberation mapping fall on the same relationship with bulge velocity dispersion as those determined from stellar dynamical measurements. The prospect is therefore that the largescale distribution of black hole masses in distant quasars may be determined through relatively straightforward measurement. Integral constraints show consistency between the total AGN luminosity density and the total volume density in SMBH contained in galaxy bulges. The strong peak of the high-luminosity quasar luminosity function at early cosmic time is consistent with the association of the build-up of SMBH through accretion and bulge formation. Alternate scenarios requiring substantial build-up of the most massive black holes at later cosmic times are more difficult to reconcile with the evolution of the LF.
\end{abstract}

\section{Gas and Stellar Dynamical Evidence for Central Black Holes}

There are two observational signatures and one requirement for the presence of a central SMBH in a galaxy nucleus. Spectra of adequate angular and velocity resolution will show rapid Keplerian rotation of gas and/or stars and a strong peak in the central velocity dispersion. The enclosed mass-to-light ratio within that region of enhanced velocities will be well in excess of that for a normal 
stellar population. The shape of the central gravitational potential can then be modeled from the motions of gas disks and/or the ensemble of stellar orbits.

For the M31 group, ground-based spatial resolution relative to the size of the central dynamically affected region actually exceeds that of HST for galaxies at the distance of the Virgo Cluster. Dressler and Richstone (1988) and Kormendy (1988) both found the strong rotational and dispersion peak signature for stellar motions in M31. They derived a best fitting central black hole mass of $7 \times 10^{7} M_{\odot}$. Dressler also pointed out the utility of the near-infrared Ca II triplet absorption feature for obtaining a clean dynamical signature that was not strongly dependent on the mix of stellar populations producing the integrated spectrum.

As is now well known, our Instrument Definition Team designed the STIS spectrograph with a performance goal of measuring galaxy nuclear dynamics (Woodgate et al. 1998, Kimble et al. 1998). The long (50") slit and 0.05" pixels were chosen to address that problem, with a balance between angular resolution and $\mathrm{S} / \mathrm{N}$ per pixel achievable on nearby galaxies.

In order to publicize the instrument after launch, NASA Headquarters requested the team to discover a new black hole. Gary Bower selected M 84, a Virgo cluster elliptical, for its suggestive kinematics in ground-based spectra, its central gas disk, and favorable radio properties. M 84 is an FR II source, with its two radio jets nearly equal in power and extent. Given the normal model of relativistic beaming, the two jets must be nearly in the plane of the sky. The axis of rotation of the inner accretion disk and probably the more extended gas disk must also lie nearly in the plane of the sky. Measured radial velocities will therefore yield nearly the full amplitude of rotation.

There is a weak AGN in the center of M 84, complicating the determination of velocity centroids. Bower et al. (1998) did derive a velocity curve for [N II], which showed both the slowly rotating outer disk and a rapidly rotating inner disk. A simple model of a cold gas disk in Keplerian rotation around a hidden dark mass, with velocities mixed through the HST+STIS PSF sampled by the 0.05 " pixels, fits the data well. That model yielded a central massive dark object of $1.5 \times 10^{9} M_{\odot}$. Neglect of line broadening (asymmetric drift, e.g., Barth et al. 2001) probably makes that value an underestimate for the mass of the SMBH. This evidence provides a direct connection between the presence of a supermassive black hole and the activity that powers quasars and radio galaxies.

Firm claim for a detection of an SMBH requires sufficient spatial resolution that other exotic high-density configurations can be ruled out. M31 is one such case; NGC 4258 provides another. It is a low-power AGN found to have strong water maser emission. Observations by Miyoshi et al. (1995) found a rapidly rotating disk of gas. The extremely high precision of radio VLBI shows a Keplerian velocity law to $1 \%$ from $0.13-0.26$ pc. The central dark mass is $3.6 \times 10^{7} M_{\odot}$. The central density is greater than $10^{9} M_{\odot} p c^{-3}$, which rules out stable alternatives to a central black hole (Maoz 1998).

Well-ordered rotating gas disks are likely to be the exception for nearnuclear configurations. Sarzi et al. (2001) found such disks in only $15 \%$ of their sample of bulges, which were already known to contain emitting gas. Currently, stellar dynamical determinations of mass are likely to be more generally reliable. Smaller elliptical galaxies and spiral bulges are rotationally supported. 
The trend of body rotation can be removed cleanly from the local rapid rotation near the nucleus. Given the run of rotation and velocity dispersion near the nucleus, stellar dynamical modeling is then required for a determination of the mass of the central Massive Dark Object. The solution of the collisionless Boltzmann equation can be derived with 2-integral or 3-integral constraints.

The most convincing case for a SMBH from stellar motions is the Galaxy itself, through proper motion measurements around Sgr A* (Genzel et al. 2000). A. Ghez et al. (1998) used the Adaptive Optics system on the Keck Telescope to obtain a time series of near-IR images. They show that the fastest transverse motion is $1350 \pm 40 \mathrm{~km} / \mathrm{s}$; the ensemble yields a black hole mass for the Milky Way of $2.6 \pm 0.2 \times 10^{6} M_{\odot}$. The central density is greater than $10^{12} M_{\odot} p c^{-3}$. Alternative clusters of compact objects would quickly evaporate, so the Massive Dark Object is demonstrated to be a Supermassive Black Hole. Emboldened by these nearby cases, we'll call other, more distant MDOs SMBH as well.

For other galaxies, the nuclear regions cannot be resolved into individual stars. The ensemble stellar motions producing the observed line profile shapes can be described by the Line of Sight Velocity Distribution (LOSVD), which can be fit parametrically with Gauss-Hermite polynomials to yield the moments of the distribution. The far-red Ca II triplet feature near $8600 \AA$ provides a robust template that is not very sensitive to modest reddening, dominant spectral type, or metallicity. The LOSVD can be derived from template matching to the broadened galaxy profiles through either the Fourier Correlation Quotient method (Bender 1990) or the Maximum Penalized Likelihood method (Merritt).

\section{The Black Hole Census and Correlation with Bulge Properties}

John Magorrian and his "Nuker Team" collaborators (1998) analyzed 36 nearby bulges with surface brightness profiles from HST images and spectra from Kitt Peak and other ground-based telescopes. They applied an axisymmetric 2integral model (i.e., constraints of only conservation of energy and angular momentum), with inclination angle, $\mathrm{M} / \mathrm{L}$, and central dark mass as the free parameters. The technique uses Maximum Likelihood to deproject the luminosity profile, calculate the gravitational potential, calculate second moments of the velocity distribution from the Jeans equation, then fit the absorption spectrum in each spatial bin. Fits can be projected as probability contours in the M/L black hole mass plane for a fixed inclination angle. Of the sample of 36 bulges, 30 contained a non-zero SMBH at high significance and 5 were consistent with a non-zero SMBH. There was only one object in which a central SMBH was ruled out, and that was distant, with a dusty core. Population statistics based on this sample showed that $97 \%$ of massive galaxies with bulges host central black holes.

They also identified a correlation, since called the "Magorrian relation", between central black hole mass and bulge luminosity. Conversion through $\mathrm{M} / \mathrm{L}$ suggested that the black hole mass was a constant fraction of total bulge mass, however with substantial scatter. The Nuker Team had initiated their investigations with HST wondering which bulges would be most likely to contain a SMBH. Within a few HST proposal cycles, the paradigm had shifted to the view that a SMBH is an integral part of every massive galaxy with a bulge. The 


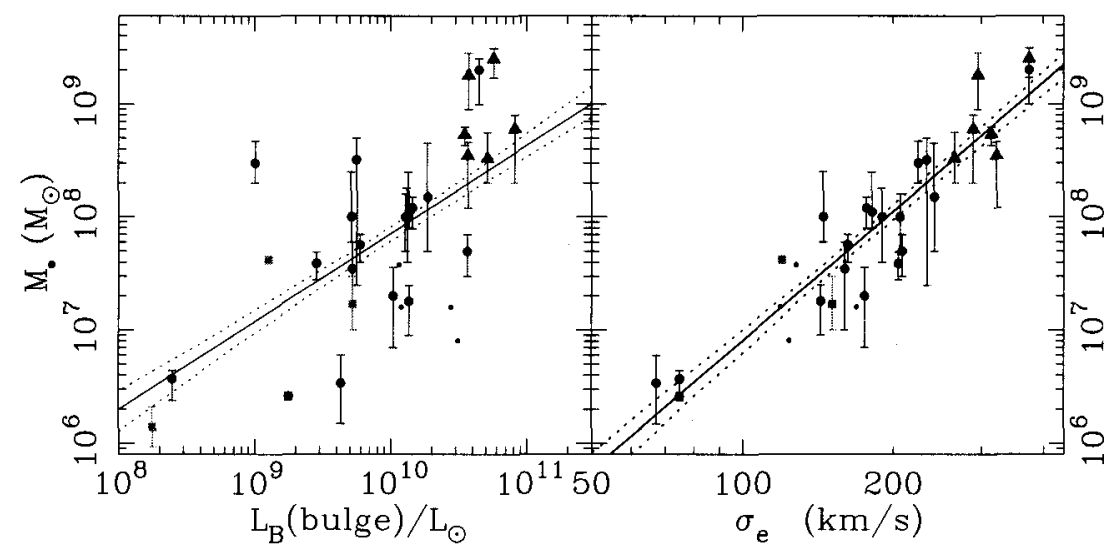

Figure 1. Left: Black hole mass vs. blue luminosity of the host bulge in solar units. Right: Black hole mass vs. luminosity-weighted velocity dispersion of the bulge.

upper limit of $\leq 10^{4} M_{\odot}$ for M 33 (Gebhardt et al. 2001, Joseph et al. 2001) argues that black holes of that scale are not found in galaxies without bulges.

An anisotropic distribution of radial orbits near the nucleus can lead 2integral models to overestimate the central mass. Richstone and Gebhardt (2002) devised a practical realization of the Schwarzschild method for finding an axisymmetric 3-integral model fit built up from pre-computed orbital families. There are now some 30 high-quality stellar kinematic determinations of central mass, a dozen good gas disks and 3 maser sources. An update to the Magorrian relation by Kormendy and Gebhardt in their thorough review (2001) gives $M_{\text {dark }}=0.78 \times 10^{8} M_{\odot}\left(L_{B, \text { bulge }} / 10^{10} L_{\odot}\right)^{1.08}$. Since $M / L \propto L^{0.2}$, the dependence implies $M_{\text {dark }} \propto M_{\text {bulge }}^{0.9}$. The dispersion in the relation is large: the rms is a factor of 2.8 , and the total range in $M_{\text {dark }}$ is two orders of magnitude at a fixed $M_{\text {bulge. }}$.

Even more significantly, Gebhardt et al. (2000) and Ferrarese \& Merritt (2000) discovered that there is a tighter correlation between black hole mass and the luminosity- weighted velocity dispersion of the bulge inside its effective radius. Gebhardt et al. (2000) and Tremaine et al. (2002) found that the scatter in the relationship may be entirely attributable to measuring uncertainties when only the best measurements are considered. That correlation is therefore more fundamental than the $M_{\text {dark }}-L_{\text {bulge }}$ correlation, although both suggest a close connection between bulge and central black hole growth.

Exceptions to the Faber-Jackson relation (like NGC 4486B) still satisfy the $M_{\text {dark }}-\sigma$ relation. We could speculate that dynamically hotter galaxies have higher surface brightnesses and smaller effective radii. If their formation 


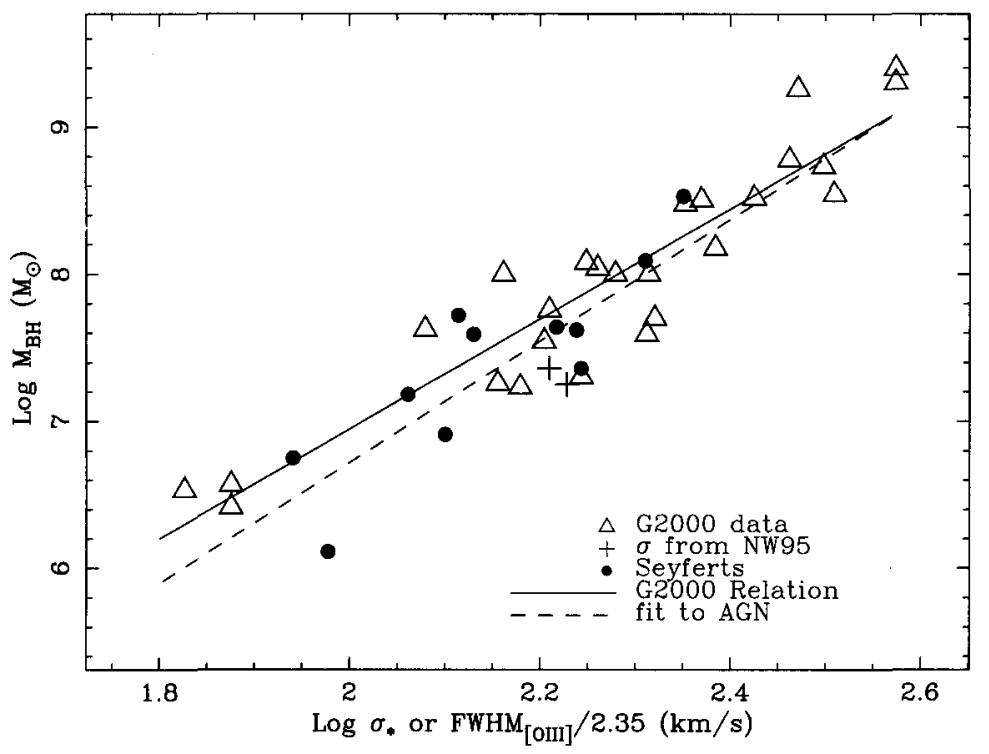

Figure 2. Black hole mass vs. host bulge velocity dispersion. Open triangles mark stellar dynamical determinations from Gebhardt et al. (2000) and the solid line marks the best fit relationship for those data. Filled circles show black hole masses derived from reverberation mapping, with the dashed line the best-fit relationship for AGN only. The agreement in zero point and slope is very close.

involved more dissipation inside their dark halos, the central black hole mass was increased proportionally. In general, the dissipative collapse and mergers that form bulges must also build the SMBH. Growing a black hole through accretion is accepted as the source of AGN luminosity. The epoch of luminous quasars is likely to be the epoch of major bulge and elliptical galaxy formation.

The current capabilities of HST and STIS allow well-constrained SMBH mass determinations out to about the distance of the Virgo Cluster for quiescent black holes. For AGN, the near-nuclear stellar dynamics cannot be measured, and the glare of the central source currently limits the ability to make direct measurements of stellar absorption in the host galaxies to all but the most modest redshifts. There is therefore active interest in finding proxies for both central dynamical measurements and probes of bulge potential. Nelson et al. (2002) and Ferrarese et al. (2001) both used the Kitt Peak 4-m to test one proxy, broad emission line width for central stellar dynamics. They used a sample of low-redshift AGN monitored for reverberation mapping of emissionline response to continuum variations. They then measured the bulge velocity dispersions for the host galaxies of those AGN. Nelson et al. found that the black hole masses from reverberation mapping and the newly measured host bulge dispersions fell along the Gebhardt et al. relation from stellar dynamics, with only a minor difference in slope. 
The agreement between the relationship based on stellar dynamical determinations and that from reverberation mapping validates the strong assumptions underlying reverberation mapping techniques:

- Line cooling from a given species lies in a relatively restricted zone at a definable effective distance from the nucleus.

- The motions of the emission-line gas are dominated by response to the gravitational potential.

- The cross-correlation derivation of time lag of response of emission-line flux to continuum variation is unambiguous.

A good correlation with moderate scatter exists between reverberation masses and photoionization model masses (Wandel, Peterson, \& Malkan 1999). The photionization derivation requires only observed spectrophotometry at one epoch. The potential therefore exists to gain a first impression of the black hole mass spectrum in AGN as a function of cosmic time, along with the typical accretion rates relative to the Eddington limit. Large-scale spectroscopic surveys such as 2DF and SDSS can in principle provide significant samples matched in luminosity for different slices of cosmic time with which the hypothesis of black hole growth with time can be tested.

\section{Implications of Integral Constraints}

Soltan (1982) and Chokshi \& Turner (1992) pointed out that the Universe should be populated today by relic black holes that were active in the quasar era with an average mass density that matches or exceeds the mass-equivalent of the energy emitted by them. Iwasawa (these Proceedings) showed us the calculation of the black hole mass density inferred from the hard X-ray background, with a local co-moving density of $6 \times 10^{5} \mathrm{M}_{\odot} p c^{-3}$. If that value is distributed according to the black hole mass vs. bulge luminosity relation through the $M / L$ of present-epoch bulges, it exceeds the local black hole mass density by a factor of only 1.4. That excellent agreement, given the uncertainty of assumptions such as bolometric corrections and accretion efficiency, gives confidence in the connection between AGN in the early Universe and today's quiescent central black holes.

It is also possible to test for general consistency that the luminous quasars of early cosmic time correspond to the most massive quiescent black holes in the current epoch. Richstone et al. (1998) presented the form of this argument, here updated with more current estimates. We assume that at turn-on quasars radiate at the Eddington limit, which specifies the black hole mass, given a measured luminosity. As summarized by Wisotzki in these Proceedings, the quasar optical luminosity function at the highest redshifts is sampled down to luminosities still higher than the characteristic luminosity, capturing only the most luminous, unobscured objects. For the faint limit of the quasar LF of $M_{B}<-26 \mathrm{mag}$, the bolometric correction of Elvis et al. (1994) gives a black hole mass of $4 \times 10^{8} M_{\odot}$. The black hole mass - bulge mass correlation then maps to $M_{B, \text { bulge }}<21.2 \mathrm{mag}$. The local galaxy luminosity function for bulges that luminous gives an integrated volume density of $10^{-5} \mathrm{Mpc}^{-3}$. 
Estimates of the high-redshift quasar LF from the first SDSS data (Fan et al. 2001) are in surprisingly good agreement with the earlier results from the transit grism survey of Schmidt, Schneider \& Gunn (1991). When combined with lower redshift data for high luminosity objects, the quasar density as a function of time shows a strong peak at a fractional lookback time of $\approx 0.85$. This "quasar epoch" had an extent in time of $\approx 10^{9}$ yrs. The peak co-moving volume density is $\approx 10^{-6} \mathrm{Mpc}^{-3}$. The luminous lifetime per object is approximated by the density of quasars / density of host galaxies $\times$ the extent of the "quasar epoch", which is $\approx 10^{8} \mathrm{yrs}$. That timescale represents $\approx 3 \mathrm{e}$-folding timescales for an accreting black hole with $10 \%$ efficiency, according to Salpeter (1964). The luminous phase of the most luminous objects is then reasonably consistent with the time required to build a SMBH. For the black hole mass - bulge mass correlation to be a consistent assumption, large bulges had to be large established by $z=2$. That assumption is also consistent with deep surveys, such as the HDF.

$\mathrm{Yu}$ and Tremaine (2002) have recently performed a detailed calculation of the integral constraints. They use the quasar LF from the 2DF (Boyle et al. 2000) to estimate the black hole mass density accreted during optically bright quasar phases. They then solve a continuity equation to relate the evolving AGN LF to the local mass density of black holes inferred from the local LF of bulges. They find that the local density of high mass black holes $\left(>10^{8} M_{\odot}\right)$ is completely accounted for by the optically luminous quasars, and that such quasars must radiate with an efficiency of $0.2-0.3$, acceptable in the model of thin-disk accretion onto a Kerr black hole. They find two options for lower mass black holes. If their growth also occurs mainly during an optically luminous stage, their accretion must occur with lower efficiency $(<0.1)$. The alternative is that they accrete with high efficiency, but that a significant fraction is obscured.

The apparent consistency of black hole mass density in luminous quasars at higher redshift and in luminous bulges locally challenges the claim that substantial growth of the most massive black holes is attributable to highly luminous obscured sources at intermediate redshifts (cf. Barger et al. 2001). The close implied connection of black hole and host bulge formation would also require substantial bulge formation or major growth in relatively recent cosmic times. Such a late build-up could require recent major mergers at a rate potentially at variance with the typical ages inferred for the majority stellar populations in luminous bulges. Alternative scenarios may mitigate the apparent conflict. Yu and Tremaine's models are consistent with lower mass, hence lower power objects accreting later or over longer time intervals. Perhaps the hard X-ray sources at intermediate redshifts are more ordinary AGNs with relatively low luminosities at OUV wavelengths. Optically faint hard X-ray sources at $z \approx 1$ observed at good ground-based resolution may still harbor optical AGNs of moderate power that are diluted by luminous host galaxies to the point of non-detection. The hard-X to optical luminosity ratio may in that case be slightly anomalous, but not necessarily require strongly Compton-thick absorption in the line of sight of intrinsically high luminosity sources.

Resolving the entangled issues of the evolution of low-mass black holes, of low- luminosity AGNs, and of the contribution of obscured sources to the total radiated accretion energy poses a challenge for the next generation of surveys. One critically important task will be to determine the optical quasar luminosity 
function on the faint side of the characteristic luminosity for $z>1$. An important anticipated step will be the deep Chandra/XMM/SIRTF/optical surveys, which should expand the multi-wavelength census and bring us closer to an unbiased count of all the manifestations of activity around SMBH.

\section{References}

Barger, A. et al. 2001, AJ, 122, 2177.

Barth, A., et al. 2001, ApJ, 555, 685.

Bender, R., 1990, a, 229, 441.

Bower, G. A., Green, R. F. et al., 1998, ApJ, 492, L111.

Boyle, B. J. et al, 2000, MNRAS, 317, 1014.

Chokshi, A. \& Turner, E. L., 1992, MNRAS, 259, 421.

Dressler, A. \& Richstone, D.O. 1988, ApJ, 324, 701.

Genzel, R. et al., 2000, MNRAS, 317, 348.

Elvis, M. et al. 1994, ApJS, 95, 1.

Fan, X. et al., 2001, AJ, 121, 54.

Ferrarese, L. \& Merritt, D., 2000, ApJ, 539, L9.

Ferrarese, L., et al. 2001, ApJ, 555, 79 .

Gebhardt, K. et al., 2000, ApJ, 539, L13.

Gebhardt, K. et al., 2001, AJ, 122, 2469.

Ghez, A. et al., 1998, ApJ, 509, 678.

Joseph, C. et al., 2001, Science, 293, 1116.

Kimble, R. A. et al., 1998, ApJ, 492, L83.

Kormendy, J., 1988, ApJ, 325, 128.

Kormendy, J. \& Gebhardt, K., 2001, in AIP Conf. Proc. 586, The 20th Texas Symposium on Relativistic Astrophysics, eds, J. C. Wheeler \& H. Martel (Melville:AIP), 363.

Magorrian, J. et al., 1998, AJ, 115, 2285.

Maoz, E., 1998, ApJ, 491, L181.

Miyoshi, M. et al. 1995, Nature, 373, 127.

Nelson, C., Bower, G. \& Green, R. F., 2002, in preparation.

Richstone, D. et al. 1998, Nature, 395, A14.

Richstone, D. \& Gebhardt, K., 2002, in preparation.

Salpeter, E. E., 1964, ApJ, 140, 796.

Sarzi, et al., 2001, ApJ, 550, 65.

Schmidt, M., Schneider, D. P., \& Gunn, J. E., 1991, AJ, 101, 2004.

Soltan, A., 1982, MNRAS, 200, 115.

Tremaine, S. et al., 2002, ApJ, 574, 740.

Wandel, A., Peterson, B. \& Malkan, M., 1999, ApJ, 526, 579.

Woodgate, B. E. et al., 1998, PASP, 110, 1183.

Yu, Q. \& Tremaine, S., 2002, MNRAS, in press. 